In: Studies in the Arts. - Neue Perspektiven auf Forschung über, in und durch Kunst und Design. Hrsg. von Michaela Schäuble und Thomas Gartmann. Bielefeld [Transcript Verlag] 2021 (= Image Bd. 195), S. 15-30.

\title{
(K)Eine Einführung in die künstlerische Forschung durch eine Musikwissenschaftlerin
}

Cristina Urchueguía

In Memoriam des Masters in Research on the Arts der Universität Bern (2011-2020)

Als Studiengangleiterin des Masters in Research on the Arts (MRA), der zwischen 2011 und 2020 an der Universität Bern angeboten wurde, durfte ich ca. hundert Künstlerinnen und Künstler verschiedenster Kunstrichtungen und unterschiedlicher Nationalitäten bei ihrer Qualifikation für ein Doktoratsstudium an der Universität Bern begleiten. Ab Herbstsemester 2019 ermöglicht eine Änderung im Reglement von swissuniversities, Absolvent"innen von Schweizer Kunsthochschulen den direkten Eintritt ins Doktorat; der MRA-Studiengang wird deshalb überflüssig. Bei allem Realitätssinn kann ich ein Quäntchen Wehmut beim Gedanken an das Ende dieses Masters nicht verhehlen. Das Tun und Denken und das ernsthafte Engagement der Studierenden haben mich beeindruckt und bereichert. Ihnen widme ich diese Gedanken.

\section{Fehleinschätzung: Musiker*innen haben keinen Verstand, Musikwissenschaftler*innen kein Gefühl}

Die Partnerschaft zwischen der künstlerischen Praxis von Musik und der wissenschaftlichen Auseinandersetzung mit Musik blickt auf eine längere Geschichte zurück. Zugegebenermaßen war der Ton im Dialog wechselhaft, aber es lässt sich nicht leugnen, dass eine aktive Beziehung existierte und weiter besteht. Obschon einem im Kontext der aktuellen Diskussion über künstlerische Forschung die Erwähnung der antiken epistemologischen 
Heimat der Musik im System der Artes Liberales immer wieder begegnet, soll dieser Kontext nicht den Ausgangspunkt meiner Überlegungen darstellen. Diese Rückgriffe erschöpfen sich in aller Regel im gelehrten Zitat, ohne methodologische Konsequenzen für die Jetztzeit nach sich zu ziehen, und wirken somit unmotiviert oder sogar kitschig. ${ }^{1}$ Jedenfalls wird die Entzweiung der Ars Musica von der Scientia, die in der Romantik als große Errungenschaft auf dem Weg zur freien Kunst galt, nicht mehr nur als Befreiung von einem kunstfeindlichen Intellektualismus gefeiert, sondern gelegentlich auch als Verlust bedauert.

Wir würden den Kern dieses Verhältnisses verfehlen, wenn wir uns der Angelegenheit essentialistisch annähern, wenn wir also nach der inhärenten Kompatibilität oder Inkompatibilität der Ansätze und Arbeitsweisen fragen würden. Unterschiede hin oder her, Musiker und Musikwissenschaftlerinnen haben sich immer irgendwie verstanden oder eben mit gegenseitigem Gewinn fürchterlich missverstanden, sie haben fruchtbar zusammengearbeitet und aufeinander gehört. Andere Kunstpraktiken und deren wissenschaftliche Pendants können nicht darauf bauen, dass Wissenschaftler*innen auch etwas skönnen<, um auf eine genauso banale wie landläufige Kunstdefinition anzuspielen.

Während die malende Kunsthistorikerin belächelt wird, gehört bei der Musikwissenschaftlerin das Musizieren zum guten Ton, und sei es (nur) ein gutgemeinter Dilettantismus beim Singen oder am Instrument. Bei Musikwissenschaftler*innen verlangt man im Gegensatz zu Kunsthistoriker"innen eine gewisse praktische Kompetenz und schreibt deren Erwerb in entsprechenden Studienplänen fest. Umgekehrt sind wesentliche Impulse für die musikwissenschaftliche Forschung von Musiker*innen ausgegangen und von diesen vorangetrieben worden. Während die Figur des sstummen

1 So Wolfert von Rahdens Statement: »Mit der Zunahme und Ausdifferenzierung von Wissenschaftsdisziplinen seit etwa der Mitte des 19. Jahrhunderts verschärfte sich die Abgrenzung der Wissenschaften von den Künsten. [...] Wen freut es nicht, wenn zwei zusammenfinden, die vom Publikum schon in der Antike als ideales Paar gesehen wurden: Schönheit und Wahrheit«, in: Wolfert von Rahden: »Wissenschaft trifft Kunst. Einführung und Dokumentation«, in: Cegenworte 23 (2010), auf: http://www.gegenworte.org/heft-23/ leseprobeheft23e.html (letzter Zugriff: 26. 8. 2019). Auch Leonardo da Vinci wird gerne als »Proto-Künstlerwissenschaftler« angeführt, vgl. Alexander Perrig: »Der Renaissancekünstler als Wissenschaftler«, in: Werner Busch/Peter Schmoock (Hg.): Kunst. Die Geschichte ihrer Funktionen, Berlin/Weinheim: Quadriga, Beltz 1987, S. 575-603. 
Künstlers`, der seine Werke für sich selbst sprechen lässt, als Klischee Karriere machte, ist das Sprechen über Musik auch im Bereich der Praxis und im Musikleben fest verankert. Sogar der Gang ins Archiv, der wissenschaftlichen Beschäftigung mit Quellen halber - nicht nur um sich Inspiration suchend der Aura des Originals auszusetzten - ist für viele, wenn auch längst nicht für alle Musiker*innen ein konstitutives Element ihrer Arbeitsweise. Danken wir dafür dem musikalischen Historismus, der u.a. auch den Nährboden für die Entstehung einer akademischen Musikwissenschaft bot.

Schließlich stellt die Personalunion beider Funktionen, also die wissenschaftlich versierte Musikerin bzw. der musikalisch-musizierende Wissenschaftler, keine Seltenheit dar. August Wilhelm Ambros (1816-1876), einer der Gründerväter der Musikwissenschaft, und Anton Webern (1883-1945) waren von Hause aus Komponisten. Letzterer ist für seine Kompositionen bekannter als für seinen Beitrag zur kritischen Edition von Heinrich Isaacs Choralis Constantinus, die 1909 als Bd. 32 der Denkmäler der Tonkunst in Österreich in Zusammenhang mit seiner Dissertation erschienen ist. Eine repräsentative Liste der Interpret*innen, die substantielle Beiträge zur musikwissenschaftlichen Forschung geleistet haben, würde den Rahmen dieses Aufsatzes sprengen.

Die Vorgeschichte der Beziehung zwischen Musik und Musikwissenschaft wirkt nach und hat der Situation der Musik im Zusammenhang der Debatte über Forschung in der Kunst eine spürbar andere Färbung verliehen als dies für die Forschung im Bereich der bildenden Künste der Fall ist, die das hauptsächliche Sorgenkind der Theoriebildung darstellt. ${ }^{2}$ Mich interessiert hier zwar vornehmlich die Musik, Seitenblicke in andere Kunstgattungen werden aber für diese Überlegungen nötig sein. ${ }^{3}$

2 Die Literatur, die Eingang in Kunsthochschulen gefunden hat und dort als kreative Tätigkeit gelehrt wird, steht in diesem Zusammenhang wieder anders da. Womöglich ist der Gang in die akademische Literaturwissenschaft für Absolvent*innen eines künstlerischen Creative Writing Studienganges naheliegender, als die Forschung an der Kunsthochschule. Jedenfalls haben sich in den Jahren 2011-2019 bei mir keine schreibendenden Künstler*innen für den Master in Research on the Arts interessiert.

3 Einen sehr brauchbaren Überblick erhält man für den mitteleuropäischen Raum in: Priska Gisler/Federica Martini: »PhD-Making is my Art Practice«, in: Julie Harboe/Federica Martini (Hg.): Swiss Artistic Research Network, 2017, S. 33-35; Jens Badura/Selma Dubach/ Anke Haarmann (Hg.): Künstlerische Forschung. Ein Handbuch, Zürich: diaphanes 2015; Julian Klein: »Was ist künstlerische Forschung?«, in: Gegenworte 23 (2010), S. 24-28, und auch in: Kunsttexte.de - E-Journal für Kunst- und Bildgeschichte Auditive Perspektiven (2011), auf: 
Die Begegnung zwischen Musiker*innen und Musikwissenschaftler*innen erfordert notwendigerweise ein vorgängiges Aushandeln des gemeinsamen Gegenstandes und gemeinsamer bzw. komplementärer oder zumindest sich nicht gegenseitig ausschließender Zielsetzungen. Die Verhandlung selbst, die auch kontroverse und dissonante Töne hervorbringen kann, betrachte ich fast schon als den eigentlichen Beitrag der Begegnung. Prinzipiell, wenn auch in wechselnder Gewandung, besteht seit der Gründung der Musikwissenschaft als Disziplin regelmäßig der notwendige Verhandlungswille, zwischen Kunstausübenden und Wissenschaftlern als wesentliche Voraussetzung wissenschaftlichen Tuns; teils schlägt sich dies in institutionellen Vereinbarungen nieder. ${ }^{4}$ Wo liegt also das Problem der künstlerischen Forschung in der Musik?

Nun habe ich mich aber lange genug dumm gestellt und dem geneigten Leser ein Paradies der Einigkeit vorgeschwärmt, in dem Friede und Freude herrschen und Eierkuchen duften, die, von heftigen Diskussionen gewürzt, nie fad schmecken. ${ }^{5}$ Wenden wir uns aber der Realität zu, denn wäre alles so

https://edoc.hu-berlin.de/handle/18452/7501 (letzter Zugriff: 9. 9. 2019). Im Bereich Musik ist vor allem das Orpheus Institut in Chent publizistisch aktiv gewesen: Jonathan Impett (Hg.): Artistic Research in Music: Discipline and Resistance: Artists and Researchers at the Orpheus Institute, Leuven: Leuven University Press 2017; Paulo de Assis: Logic of Experimentation: Rethinking Music Performance through Artistic Research, Leuven: Leuven University Press 2018.

4 Zu den prominenten institutionalisierten Orten der Zusammenarbeit seien hier nur die Basler Schola Cantorum, 1933 explizit als »Lehr- und Forschungsinstitut« gegründet, auf https://www.musik-akademie.ch/schola-cantorum-basiliensis/de/uber-uns/geschichte. html (letzter Zugriff: 30. 7. 2019) erwähnt, sowie das Orpheus Institut in Chent, das seit 1996 Advanced studies \& research in Music anbietet. Dazu wäre eine ganze Reihe von Universitäten in USA, Lateinamerika, Deutschland und Österreich zu nennen, in denen musikalische Ausbildung im universitären Kontext stattfindet.

5 Darla Crispin schlägt in ihrem Beitrag »Musik«, in: Jens Badura/Selma Dubach/Anke Haarmann (Hg.): Künstlerische Forschung. Ein Handbuch, Zürich: diaphanes 2015, S. 31-33, andere Töne an: »Das akademische Musikstudium als eigenständiges Fach wiederum entstand im neunzehnten Jahrhundert, was sich in der Prägung des Begriffs >Musikwissenschaft Anfang des zwanzigsten Jahrhunderts niederschlug. Einzelne Musiker mochten zwar zwischen den beiden Welten hin und her wandern, aber auf institutioneller Ebene gab es kaum Überschneidungen.«(S. 31). Leider ist die Autorin über die historischen Zusammenhänge nur sehr ungenau informiert. Eine der wenigen Polemiken findet sich als Satire in: Therese Bruggisser-Lanker: »Schöne neue Kunstwelt«, in: Schweizer Musikzeitung, 06/2011, S. 21, auf: https://www.musikzeitung.ch/de/smz/printarchiv.html?q=bruggisser\#. XYHsXndu]aQ (letzter Zugriff: 9. 9. 2019). 
einfach wie oben beschrieben, würden nicht Monographien und Sammelbände zur künstlerischen Forschung mehrere Regalmeter füllen. Die Problematik ist komplizierter.

\section{Fehleinschätzung: Es geht um die Sache}

Die heute mit großer Intensität geführte Debatte über künstlerische Forschung hat erst Ende des 20. Jahrhunderts und verstärkt zu Beginn des 21. Jahrhunderts Fahrt aufgenommen. Der Anlass war nicht Leidensdruck von Seiten wissenschaftlicher Entscheidungsträger ob etwaiger $\mathrm{Zu-} \mathrm{oder}$ Missstände, vielmehr üben sich die Vertreter*innen der universitären Musikforschung bei der inhaltlichen Debatte, sieht man von vereinzelten Stellungnahmen - nicht selten Unkenrufen über den bevorstehenden Weltuntergang -, vorwiegend in edler Zurückhaltung. ${ }^{6}$ Befeuert wurde die Diskussion von den Kunsthochschulen, weil die Entwicklungen, die zur Debatte führten, in erster Linie Institutionen der Kunstausbildung vor grundlegende Reformen stellten; die Universitäten verspürten bezüglich der Kunstforschung lediglich die Kollateraleffekte. Der Grund für die extensive und intensive Auseinandersetzung mit künstlerischer Forschung liegt in einer 1999 begonnenen, flächendeckenden Veränderung innerhalb der akademischen Großwetterlage im europäischen Hochschulsystem: dem Bologna-Prozess. ${ }^{7}$ Es geht also nicht in erster Linie um die Sache, sondern um eine Verortung im System und folglich muss eine Analyse der Debatte zunächst systemisch und dann inhaltlich vorgehen.

Die Situation von Ausbildung und Tätigkeit von Musiker*innen und $\mathrm{Mu}-$ sikwissenschaftler*innen in der ganzen Welt ist zu divergent, um ein globales Bild entwerfen zu können. Ich konzentriere mich daher auf die Schweiz und werfe nur Seitenblicke woanders hin. Diese Beschränkung verringert

\footnotetext{
6 Selbst Ulrich Konrad, der 2007 in seinem Aufsatz »Ars - MUSICA - scientia. Gedanken zu Geschichte und Gegenwart einer Kunst und ihrer Wissenschaft«zur richtigen Zeit das richtige Thema hätte behandeln können, versäumte die Gelegenheit, sich der Forschung an Kunsthochschulen zu widmen, sie kommt in seinem Bild von Musikwissenschaft nicht vor, in: Laurenz Lütteken (Hg.): Musikwissenschaft. Eine Positionsbestimmung, Kassel: Bärenreiter 2007, S. 20-39.

7 Kathrin Busch/Dieter Lesage (Hg.): A Portrait of the Artist as Researcher: The Academy and the Bologna Process, Antwerp: MUHKA 2007.
} 
die Komplexität zwar, aber trotzdem wird es nicht einfach. Nicht einmal im europäischen Hochschulraum, der von dem transnationalen BolognaProzess in ein Latifundium vereinheitlichter Studiengänge und -abschlüsse sowie ungehemmter Studienmobilität verwandelt werden sollte, kann von Gleichschaltung die Rede sein. Das Ergebnis der ersten Phase des Prozesses ist eine Standardisierung der Abschlüsse Bachelor und Master bei gleichzeitiger polysemischer Anreicherung dieser durch die Kontamination mit dem Erbe der ehemaligen akademischen Systeme. Nach der Maxime »so viel wie nötig und so wenig wie möglich « haben die verschiedenen nationalen Systeme versucht, möglichst viele ihrer Charaktereigenschaften in das neue System hinüber zu retten. Umetikettierungen einerseits und das Ausreizen der Optionen andererseits führten zur tatsächlichen Mannigfaltigkeit in der scheinbaren Einheit. Mehr wäre am Beginn eines solchen Prozesses in einem so sensiblen Bereich wie der Hochschulpolitik utopisch gewesen, es ist aber ein unabgeschlossener Prozess, ein work in progress.

Die Kunstausbildungsstätten standen vor einer substantielleren Herausforderung als Universitäten, obschon die Umsetzung auch bei den Universitäten ein Mammutunternehmen war. Denn zum einen gingen frühere internationale Homogenisierungsbestrebungen im europäischen Hochschulsektor explizit von Universitäten aus: Berufsbildende und kunstausbildende Schulen wurden erst kurz vor der Einführung des Bologna-Prozesses und nur implizit berücksichtigt. ${ }^{8}$ Zum anderen, weil die Ausgangslage in den verschiedenen Ländern auch unterschiedlich war. In England

8 Liest mandas»Gesetzzu dem Übereinkommen vom 11. April1997überdie Anerkennungvon Qualifikationen im Hochschulbereich in der europäischen Region«, Bundesgesetzblatt, Jahrgang 2007, Teil II, Nr. 15, ausgegeben zu Bonn am 22. Mai 2007, hier S. 714, auf: www. bgbl.de/xaver/bgbl/start.xav?startbk=Bundesanzeiger_BCBI\&jumpTo=bgbl207s0712.pdf (letzter Zugriff: 9. 9. 2019), S. 712-732, so fällt bei der Liste der Konventionen des Europarats und der UNESCO, auf die die Konvention gründet, auf, dass nur Universitäten erwähnt werden: z. B. Europäische Konvention über die Cleichwertigkeit der Reifezeugnisse (Diplomas leading to Admission to Universities) (1953), Europäisches Übereinkommen über die allgemeine Gleichwertigkeit der Studienzeiten an Universitäten (1990). Die Sorbonne-Erklärung von 1998 hingegen erweitert die Überlegungen auf den Bildungsbereich im Allgemeinen im Kontext der Verpflichtung zu »lebenslangem Lernen« aus: »Wir sehen uns auch einer Zeit grundlegender Veränderungen im Bildungsbereich und am Arbeitsplatz gegenüber, einer Diversifizierung der Berufsbildung, in der lebenslanges Lernen zu einer ganz klaren Verpflichtung wird.«, in: »Sorbonne-Erklärung«(1998), auf: https://www.sbfi.admin. ch/sbfi/de/home/hs/hochschulen/bologna-prozess.html (letzter Zugriff: 9. 9. 2019), S. 1. 
studierten Musiker*innen an einer Universität, in Spanien führten die Conservatorios Superiores den Status einer höheren Sekundarschule, in der Schweiz galten die Konservatorien als Höhere Fachschulen, um nur einige dieser Vorgeschichten zu beschreiben. Der Handlungsbedarf war so offenkundig wie das Konfliktpotenzial.

Die Situation in der Schweiz wurde von einer landesspezifischen Hochschulstrategie noch zusätzlich verkompliziert. Ehemals autonome Konservatorien und Höhere Fachschulen verschiedener Kunstrichtungen - Bildende Kunst, Musik, Theater, Tanz, Literatur - und sogar Berufe wie Restaurierung und andere kreative Tätigkeiten wie Design, die sich nicht unbedingt als künstlerische Berufe verstanden, wurden institutionell fusioniert und unter das Dach regionaler Fachhochschulen geparkt, die wiederum auch aus der Zusammenlegung vieler anderer Höheren Fachschulen entstanden. ${ }^{9}$ Man will sich nicht vorstellen, wie viele Meetings für diese Fusionspläne nötig gewesen sind. Die dergestalt ins Leben gerufenen Megalopolen der Berufsbildung wurden zur Fachhochschule aufgewertet und erhielten folglich einen Forschungsauftrag. Die Forschungstätigkeit war somit akademisch gesehen eine Imposition up to bottom, für deren Erfüllung viele der neugegründeten Fachhochschuldepartemente weder auf ein Selbstverständnis noch auf eine Tradition zurückgreifen konnten. Das eigens für die Fachhochschulen ins Leben gerufene SNF Förderprogramm DO REsearch, kurz DORE, ermöglichte erste Versuche in einer allseits beliebten Gattung: dem Fördergesuch. ${ }^{10}$

Der Prozess zielte nicht auf eine Revolution der Kunstausbildung, vielmehr galt es, Forschung, Entwicklung und Innovation synergetisch mit der traditionellen Berufsausbildung zu koppeln. Idealiter sollten Fachhochschulen das durch universitäre Grundlagenforschung gewonnene Wissen

9 Diese hochschulpolitische Entscheidung führte etwa zur Entstehung der Berner und Zürcher Fachhochschulen und der Fachhochschule Nordwestschweiz, inklusive Hochschule der Künste Bern, Zürcher Hochschule der Künste und Basler Musikakademie inklusive Musikhochschule Basel und Schola Cantorum Basiliensis, um nur einige der größten zu nennen.

10 Ernüchternd spricht Cornelia Sollfrank darüber in:»A pervert's guide to Artistic research«, in: Judith Siegmund (Hg.): Wie verändert sich Kunst, wenn man sie als Forschung versteht?, Bielefeld: transcript 2016, S. 94: »Bei einer Verteilung der Verpflichtungen von 50 Prozent Forschungstätigkeit und 50 Prozent Lehre bestand die Forschungstätigkeit in meinem Fall vorwiegend nicht etwa in der Realisierung von Kunstprojekten, sondern im Erarbeiten von Anträgen, Erstellen von Evaluierungen und Peer-to-peer-Reviews sowie der Mitarbeit in diversen Forschungsgremien.« 
forschend anwenden und somit innovative Produkte entwickeln, die der Gesellschaft unmittelbare Dienste leisten: vom optimierten Rollstuhl für die Krankenpflege bis zur bahnbrechenden Säge für die Holzverarbeitung. Stellvertretend lasse ich die Netzkünstlerin Cornelia Sollfrank zu Wort kommen, die sich der Situation ohne Scheuklappen und Euphemismen stellt:

„Über das Verhältnis von Kunst, Forschung und Wissen kann man nicht sprechen ohne anzuerkennen, dass der unmittelbare Anlass dafür hochschulpolitische Entwicklungen sind, in denen es weniger darum geht, Grundlagenforschung zu ermöglichen oder Freiräume für neue Formen der Wissensproduktion zu schaffen, als vielmehr administrativ vorgegebene Formate und Standards flächendeckend einzuführen - auch für die Kunst. « ${ }^{11}$

Dass sich die Kreativität der Kunst von der landläufigen Produkt- und Dienstleistungsinnovation genauso unterscheidet wie das wissenschaftliche Selbstverständnis der Geisteswissenschaften sei hier nur als tiefsitzende Problemlage erwähnt, die den epistemologischen Rahmen der Debatte über Forschung an Kunsthochschulen wesentlich mitprägt. ${ }^{12}$

Gleichwohl konnte man auf Erfahrungen in anderen Ländern aufbauen. Großbritannien und Australien, aber auch die Niederlande und Schweden hatten Jahrzehnte früher die Einbindung der Künste in den Forschungsap-

11 Ebd., S. 87.

12 Siehe dazu etwa die Broschüre des SBFI (Hg.): »Forschung und Innovation in der Schweiz 2016« (2016), auf: www.sbfi.admin.ch/f-i_bericht (letzter Zugriff: 23. 9. 2019). Zentrales Anliegen ist der Transfer von durch Forschung gewonnenes Wissen und Innovation im Bereich von Technologie auf produzierende Unternehmen. Es wird dabei eine Unterscheidung zwischen Grundlagenforschung und angewandter Forschung, die Kunst und Geisteswissenschaften prinzipiell außen vor lässt, gemacht. Die SACW antwortete auf diese Broschüre mit dem Sammelband: Innovation. Anregungen/Impulse aus den Geistes- und Sozialwissenschaften. Ein Werkbericht. Bern: SACW 2018, auch auf: https://sagw.ch/fileadmin/ redaktion_sagw/dokumente/Publikationen/Berichte/Innovation_Werkstattbericht.pdf (letzter Zugriff: 23. 9. 2019), in dem die Ausgrenzung der Geistes- und Sozialwissenschaften angeprangert wurde. Im Diskurs der Künstlerischen Forschung hat Henk Borgdorff auf die problematische Dichotomie zwischen Grundlagenforschung und angewandter Forschung aufmerksam gemacht: »The debate on research in the arts« (2006), auf: www. ips.gu.se/digitalAssets/1322/1322713_the_debate_on_research_in_the_arts.pdf (letzter Zugriff: 1. 2. 2015), S. 9. 
parat und die Integration der Forschung in die Kunstausbildung vollzogen. ${ }^{13}$ Auch Deutschland führte mit der Bologna-Reform einige Musikhochschulen in Universitäten über und erteilte, wenn auch nur in begrenztem Maße, das Promotionsrecht, eine sheilige Kuh<, die in der Schweiz noch immer ein Monopol der universitären Hochschulen darstellt. Die ersten Reaktionen von beiden Seiten, Musikhochschulen und Universitäten, waren nicht besonders freundlich. Hier nur als Beispiel eine davon: Als am Würzburger Institut für Musikwissenschaft, an dem ich damals promovierte, die bevorstehende Einführung des Doktorats an der Würzburger Musikhochschule bekannt wurde, ca. 1998-1999, machte die unschöne Bezeichnung des >Doktor Klimp< die Runde, mit der man das neue Doktorat herabzuwürdigen trachtete. Die universitäre Musikwissenschaft sah den Untergang des Abendlands wie ein Pleitegeier über ihre heile Welt kreisen, die Kunsthochschulen fürchteten im Gegenzug die kunsttötende Akademisierung. ${ }^{14}$ Von heute auf morgen galt es, aus der Opposition zwischen Kunst und Wissenschaft ein Binom zu zaubern.

13 Sehr lesenswert ist in diesem Zusammenhang der Beitrag von Torsten Kälvemark über die Einführung von Forschung in den schwedischen Kunsthochschulen. Torsten Kälvemark: »University Politics and Practice-Based Research«, in: Michael Biggs und Henrik Karlsson (Hg.): The Routledge companion to research on the Arts, Stockholm: Routledge 2011, S. 3-23.

14 Siehe Borgdorff 2006, S. 2. Folgendes Cespräch fasst die Verwerfungen und Schwierigkeiten bei der Umsetzung in der Hamburger Hochschule bündig zusammen und benennt die Vorurteile zur Beziehung von Kunst und akademischer Forschung: Holger Tiedemann und Cornelia Sollfrank: »ausnahmsweise freie kunst. Ein Gespräch« (2008), auf: http:// artwarez.org/95.o.html (letzter Zugriff: 30. 7. 2019): »[Cornelia Sollfrank:] Und ich finde den Widerspruch interessant, dass manche Kunsthochschulen auf der einen Seite sagen, wir wollen nicht quantifizierbar und qualifizierbar ausbilden, aber dann doch das Promotionsrecht haben. Spätestens an dieser Stelle müssten sie doch in der Lage sein, genau zu begründen, warum sie das wollen und was es sein soll. Um es etwas polemisch auszudrücken: Ist eine Promotion für einen echten Künstler nicht sogar schädlich? Zuviel intellektuelle Betätigung könnte womöglich die Reinheit des Cefühls und des Ausdrucks beeinträchtigen. [...]. Sehr viel an dieser ganzen Diskussion kommt mir sehr unausgegoren und absurd vor, auf allen Seiten, aber dass die Kunsthochschulen und Künstler es immer wieder schaffen, ihr >Anderssein « zu perpetuieren, ist auf jeden Fall eine tolle Leistung." Zu den Effekten der Forschungstätigkeit auf die Kunst sei folgender Titel empfohlen: Judith Siegmund (Hg.): Wie verändert sich Kunst, wenn man sie als Forschung versteht?, Bielefeld: transcript 2016. Interessant ist hier der Versuch einer Cleichsetzung von Kunst und Forschung. 


\section{Der Umgang mit dem Forschungsmandat: I. Vorgeschichte}

Die Umsetzung des Forschungsmandats verlief auf unterschiedlichen Fronten mit jeweils unterschiedlichen Taktiken. Die praktische Front, was also in den Kunsthochschulen unternommen wurde, um Forschung in die Organigramme und in das System der Hochschulen zu implementieren, wurde von einer wahren Flut an theoretischer Reflexion und hochschulpolitischer Aktivität sekundiert. Ich konstatiere aus der Vogelperspektive und mit gebührender Vorsicht einen Mangel an Koordination zwischen den Stoßrichtungen. Gleichzeitig sehe ich mich außer Stande, diese Aktionsfelder in einer repräsentativen Weise Revue passieren zu lassen. Denn die unübersehbar komplexen akademischen Entscheidungen, die auf der operativen Ebene an unzähligen Standorten getroffen und umgesetzt worden sind, fanden ihren Niederschlag in Regelwerken, die im Gegensatz $\mathrm{zu}$ den übergeordneten europäischen Vereinbarungen nicht für die Öffentlichkeit gedacht waren. Der Umsetzungsprozess verdient eine genaue quellenbasierte Beurteilung, bei der man sicherlich Tonnen von Reglementen, Vereinbarungen und Studienplänen wälzen müsste; alles in allem ein prädestiniertes Objekt für eine interdisziplinäre wissenschaftliche Arbeit. Hier berufe ich mich lediglich auf meine eigene Anschauung und auf die im Zusammenhang des Masters in Research on the Arts und der Graduate School of the Arts gewonnenen Erkenntnisse.

Der Ansturm der Theorie setzt wiederum an zwei Stellschrauben an, einerseits der epistemologischen Positionierung der Kunst im System des Erkenntnisgewinns in Abgrenzung zum wissenschaftlichen Diskurs, andererseits an der Einflussnahme auf die Institutionen, die wissenschaftliche Qualitätsstandards festlegen. Prophetisch klingt Dennis Strands vor über 20 Jahren geäußertes Misstrauen gegenüber Versuchen, den »mainstream of creativity arts activities« den Stempel der Wissenschaftlichkeit überzustülpen: »Attemps to force mainstream creative arts activities into the mould of scientific research has led to semantic arguments that often have not been particularly helpful «. ${ }^{15}$ Dieses Reserve gegenüber Wissenschaftlichkeit schlägt sich in der Wahl der Terminologie nieder: Die Debatte in den Kunst-

15 Dennis Strand: Research in the creative arts. Australia. Department of Employment, Education, Training and Youth Affairs. Evaluations and Investigations Program, Canberra: Department of Employment, Education, Training and Youth Affairs 1998, S. xv-xvi. 
hochschulen handelt nicht von Wissenschaft, sondern von Forschung; der wissenschaftlichen wird eine andere, nämlich die künstlerische Forschung gegenübergestellt. Was aber künstlerische Forschung ist und wie diese sich bei der Wissensproduktion und -vermittlung spezifisch verdient machen kann, wurde bisher nicht brauchbar festgelegt. Die an den Kunsthochschulen für die Forschung Verantwortlichen haben in aller Regel mit der etablierten universitären Forschung notorisch gefremdelt, der fehlende Dialog geschah, wie mir scheint, zum Nachteil beider.

Die prinzipielle Abneigung gegen wissenschaftliche Herangehensweisen, die sich in seiner extremsten Form in der Erklärung "Kunst ist Forschung « äußert, kann ebenso zur Selbstverblendung führen, wie bedingungslose Unterwerfung. ${ }^{16}$ Die Hypostase einer Gleichsetzung von Kunst und Forschung ist insofern problematisch, als es bei Erkenntnis auch um eine Anreicherung geht, also um die Möglichkeit, neue Forschungsinteressen auf frühere Forschungsergebnisse aufzubauen. Es ist die Vermittlung von Erkenntnissen und die Ermittlung von Relevanz und Pertinenz in einem qualitätsgeprüften Forschungsdiskurs, die der künstlerischen Forschung die größte Mühe macht. Wie beim fallenden Baum, der nur dann gefallen ist, wenn es jemand beobachtet hat, existiert Wissen nur dort, wo es wahrgenommen und verstanden wird.

Wenn Julian Klein, seines Zeichens Komponist, Theaterregisseur und seit 2018 Präsident der Gesellschaft für künstlerische Forschung der BRD auf die Frage "Was ist künstlerische Forschung?", die freche Gegenfrage hinpfeffert »Sorry, die Frage ist falsch gestellt, Good man (1978). Wir sollten fragen: Wann ist Forschung künstlerisch? « ${ }^{17}$, wundert man sich über den aggressiven Unterton, zumal der Autor sich mit dieser Reaktion in die unbequeme Situation hineinmanövriert, auf fünf Seiten eine Definition des Künstlerischen und der künstlerischen Erfahrung beisteuern zu müssen.

Viele Texte, die ein spezifisches künstlerisches Wissen, eine eigene künstlerische Episteme behaupten, wirken auf mich wie eine Trotzreaktion. Mir scheint, dass einige Kunsthochschulen die Wortführung bei Philo-

16 Siehe: Serge Stauffer: »Kunst als Forschung«, in: Gerhard Johann Lischka/Hansjörg Mattmüller (Hg.): Cenie gibt's: die siebziger Jahre an der F \& F Schule für Experimentelle Gestaltung, Frankfurt a.M.: Betzel 1981, S. 61-93.

17 Julian Klein: »Was ist künstlerische Forschung?«, in: Gegenworte 23 (2010), S. 24-28, auch in: Kunsttexte.de - E-Journal für Kunst- und Bildgeschichte Auditive Perspektiven (2011), auf: https://edoc.hu-berlin.de/handle/18452/7501 (letzter Zugriff: 9. 9. 2019). 
soph"innen und Kulturtheoretiker*innen in Auftrag gaben, die sich weder von der Anschauung der tatsächlichen künstlerischen Tätigkeit anleiten ließen, noch einen offenen Austausch mit der universitären Forschung suchten. Stattdessen nutzten sie diese Bühne für philosophische Vorlesungen über Kunstforschung, die von Adorno über Derrida, Heidegger und Nietzsche bis Žižek nichts ausließen, aber die Künstler*innen selbst kaum zu Wort kommen ließen. ${ }^{18}$

Auch Kathrin Busch bleibt uns in ihrem Sammelband Anderes Wissen eine Begründung der Behauptung einer fundamentalen Verschiedenheit zwischen künstlerischem Wissen, dem anderen Wissen und Wissen - womöglich eigentliches Wissen? -, schuldig. Ihre Behauptung lautet lapidar:

»Andersheit kommt dem künstlerischen Wissen zuallererst in Absetzung zur wissenschaftlichen Erkenntnisproduktion zu, insofern es sich um eine eigenständige Form der Wissensbildung handelt, die mit anderen Forschungsverfahren, Darstellungsformen und auch Rezeptionsweisen als die Wissenschaften operiert. Das künstlerische Forschen führt zu einem gegenüber den Wissenschaften andersgearteten Wissen. ${ }^{19}$

Hierbei wird die Vielfalt an Forschungsansätzen, der Reichtum an Wissenstheorien und die Uneinheitlichkeit der Weisen von künstlerischer Forschung bzw. von Kunstdefinitionen an sich ignoriert: Dieses Zitat setzt eine Wissenschaft, eine Kunst voraus, und der restliche Text beißt sich, im Versuch einer Nuancierung die Zähne aus. Allein schon die Wortwahl, die manichäische Opposition zwischen (tendenziell gutem) »künstlerischem Wissen « und (böser, weil ökonomisierter) "wissenschaftlicher Erkenntnisproduktion", hinterlässt einen schalen Beigeschmack. Busch selbst war sich zudem nicht immer im Klaren, ob ein Wissen der Künste überhaupt existiert:

18 Ein Beispiel wäre hier: Elke Bippus‘ Einleitung zu »Kunst des Forschens. Praxis eines ästhetischen Denkens«, in: Elke Bippus (Hg.): Schriftenreihe des Instituts für Gegenwartskünste, ZHdK, Bd. 4, Zürich und Berlin: diaphanes 2009, S. 7-24.

19 Kathrin Busch: Anderes Wissen, Paderborn: Fink 2015, S. 11. Der Diskurs einer eigenen Episteme künstlerischen Wissens wird in einer ganzen Reihe von Publikationen reflektiert, hier nur eine: Eva-Maria Jung: »Die Kunst des Wissens und das Wissen oder Kunst. Zum epistemischen Status der künstlerischen Forschung«, in: Siegmund 2016, S. 23-44. 
»Die Frage danach, was skünstlerische Forschung`sei, bleibt bis heute unbeantwortet. Im Zentrum der Auseinandersetzung steht die Frage, welche Relevanz begriffliches Wissen und wissenschaftliche Methoden für die künstlerische Forschung haben. Strittig ist, ob die künstlerische Forschung Eigenständigkeit gegenüber der Wissenschaft beanspruchen kann und inwiefern sich das Wissen der Künste von dem wissenschaftlichen Wissen unterscheidet. ${ }^{20}$

Die Dämonisierung traditioneller Wissenschaft durch die Theoretiker"innen der Debatte bedient sich nolens volens ausgerechnet jenes wissenschaftlichen Duktus, den die Autor*innen als akademisch ausgebildete Philosoph"innen oder Kulturtheoretiker*innen von Beginn an gelernt haben. ${ }^{21}$ Ich hätte entgegnet: »Auch wenn euer Auftraggeber jetzt eine Kunsthochschule ist, hat Euch die Wissenschaft wirklich nichts getan.«

\section{Der Umgang mit dem Forschungsmandat: II. Taten statt unanswered questions}

Bodenständig, ja, hemdsärmelig ging es an der institutionellen Front zu. In seiner bereits 1998 erschienenen Broschüre Research in the creative arts. Australia im Auftrag des Departements für Arbeit, Bildung, Berufsausbildung und Angelegenheiten der Jugend, einer Inkunabel dieser Debatte, bringt Dennis Strand die Problematik auf den Punkt:

»A key issue for this study is the place of the creative arts in the Composite Index, a mechanism which determines how the Research Quantum is distributed. ${ }^{22}$

20 Kathrin Busch: »Künstlerische Forschung«, in: Stephan Günzel/Dieter Mersch (Hg.): Bild. Ein interdisziplinäres Handbuch, Stuttgart und Weimar: Metzler 2014, S. 453.

21 Ich verweise auch auf Publikationen wie Martin Tröndle/Julia Warmers (Hg.): Kunstforschung als ästhetische Wissenschaft. Beiträge zur transdisziplinären Hybridisierung von Wissenschaft und Kunst, Bielefeld: transcript 2012; oder Anke Haarmann: »Künstlerische Praxis als methodische Forschung« (2011), auf: www.dgae.de/wp-content/uploads/2011/09/ Haarmann.pdf (letzter Zugriff: 20. 3. 2017).

22 Strand 1998, S. 85. 
Es geht also nicht um Inhalte oder Fragestellungen, Methoden oder Wissensdefinitionen, sondern um die Kriterien für die Verteilung der Forschungsressourcen. Der Australische Composite Index wäre hierzulande das von der OECD gepflegte Frascati Manual, in dem die verbindlichen Kriterien für die Bewertung und Messung von wissenschaftlichen, technologischen und innovationsfördernden Aktivitäten festgesetzt werden. ${ }^{23}$

Aus einer hochschulpolitischen Sicht ging es den Kunsthochschulen um nichts Anderes als Gleichbehandlung oder sogar um Gerechtigkeit. Wenn wir schon forschen müssen, dann bitte unter Bedingungen, die für die Qualität unserer Kernbeschäftigung relevant sind, so könnte man das Argument zuspitzen. Die europäischen Stakeholder haben dafür auch von einem Instrument Gebrauch gemacht, das sich für politische Tätigkeit eignet: Lobbyismus. Neue nationale Vereine wie SARN (Swiss Artistic Research Network ${ }^{24}$ ) wurden gegründet, zusammen mit bestehenden Vereinen wie der seit 1990 operierenden ELIA (European League of the Institutes of the Arts ${ }^{25}$ ) bündelten sie die Interessen der Kunsthochschulen national und international, um bei hochschulpolitischen Initiativen als Ansprechpartner*innen anerkannt zu werden.

Die »Florence Principles on the Doctorate in the Arts«, die im Auftrag von ELIA 2016 formuliert wurden, veranschaulichen den Zustand der Diskussion auf der institutionellen Ebene in allgemeingehaltener, abwägender und einschließender Weise. ${ }^{26}$ Die Autor*innen stellen jedoch etwas zu optimistisch eine ursächliche Beziehung zwischen der »inclusion of sartistic research in OECDs' Frascati Manual und der Einsetzung ihrer Arbeitsgruppe her. Tatsächlich zeigt ein Blick in das genannte Frascati Manual von 2015, dass dort »artistic research « nicht vorkommt, stattdessen unterscheidet das

23 Frascati Manual: »The Measurement of Scientific, Technological and Innovation Activities. Guidelines for Collecting and Reporting Data on Research and Experimental Development« (2015), auf: https://dx.doi.org/10.1787/9789264239012-en (letzter Zugriff: 9. 9. 2019).

24 SARN: Swiss Artistic Research Network (gegründet 2011), auf: https://sarn.ch/ (letzter Zugriff: 9. 9. 2019).

25 ELIA: European League of the Institutes of the Arts, auf: https://www.elia-artschools.org/ (letzter Zugriff: 9. 9. 2019).

26 European League of the Institutes of the Arts (ELIA) (Hg.): »The ^Florence Principles on the Doctorate in the Arts« (2016), auf: http: www.elia-artschools.org/userfiles/File/ customfiles/1-the-florence-principles2016112 (letzter Zugriff: 9. 9. 2019). 
Handbuch im Abschnitt »R\&D and artistic creation « zwischen »research for the arts, research on the arts and artistic expression $« .{ }^{27}$ Gegenüber der Fassung von 2002 gab es aber doch eine entscheidende Aufwertung der Kunst im System der geisteswissenschaftlichen Forschung. Während das Frascati Manual 2002 »artistic >research« explizit als Betätigungsfeld für Forschung und Entwicklung ausschließt ${ }^{28}$, wird 2015 die Forschung für und an Kunst anerkannt, und nur noch der künstlerische Ausdruck selbst ausgeklammert:

»2.67 Artistic performance is normally excluded from R\&D. Artistic performance fails the novelty of R\&D as they are looking for a new expression, rather than for new knowledge. Also, the reproducibility criterion [...] is not met. $^{29}$

2015 hatte man Grund zur Freude, aber nicht wegen einer vollumfänglichen Integration künstlerischer Forschung im Sinne künstlerischer Praxis in den Kanon der Forschung.

Uneingedenk etwaiger Verwerfungen verfolgten Netzwerke wie das Swiss Artistic Research Network (SARN) ihre Tätigkeit, ohne großen Wirbel darum zu machen. Die Publikationen, die aus den jährlichen Treffen hervorgegangen sind, zeugen von systematischer Arbeit an Grundlagen, deren realistische Bescheidenheit wohltuend ist. Das Netzwerk befragte forschende Künstler*innen zu ihren Sorgen ${ }^{30}$, es beschäftigte sich mit tatsächlichen Schwierigkeiten, wie zum Beispiel der Frage nach der Sprache und den Publikationswegen ${ }^{31}$, realen Problemen, die nicht nur denjenigen, die neu in der Forschung sind, Kopfzerbrechen bereiten.

All diese politischen und theoretischen Hintergründe wurden mir erst bewusst, als ich bei der Vorbereitung einer Einführungsveranstaltung für

27 Frascati Manual 2015, S. 64f. siehe Anm. 23.

28 Frascati Manual: Proposed Standard Practice for Surveys on Research and Experimental Development« (2002), auf: https://read.oecd-ilibrary.org/science-and-technology/frascati-manual-2002_9789264199040-en\#page1 (letzter Zugriff: 23. 9. 2019), S. 67: „Other humanities [...] excluding artistic >research s of any kind.»

29 Frascati Manual 2015, S. 65.

30 Flavia Caviezel/Markus Schwander: Eigthy-Seven Questions on Artistic Research, Basel: SARN 2015.

31 Barnaby Drabble/Federica Martini (Hg.): Publishing artistic research, Basel: SARN 2014. Lucie Kolb et al. (Hg.): Artistic Research: The Language Issue, Basel: SARN 2014. 
die Graduate School of the Arts 2015 und 2017 eingehendere Recherche betrieb. Ich war etwas verwirrt, weil meine Arbeit mit den Studierenden im Master in Research on the Arts von dieser Vorgeschichte weitgehend unbehelligt geblieben war und auch blieb. Vielmehr hatte eine glückliche Fügung, und zwar der proaktive Kooperationswille zwischen der Philosophisch-historischen Fakultät der Universität Bern und der Leitung der Hochschule der Künste Bern einzigartige Rahmenbedingungen für die inhaltliche Arbeit hergestellt. Einerseits standen die Studierenden nicht unter Beweiszwang; die Immatrikulation an der Universität machte sie zu gleichberechtigen Studierenden, andererseits fanden sie bei ihren Betreuer*innen in aller Regel offene, ja neugierige Ohren für ihre Interessen und Anerkennung für ihre Kompetenzen. Die Verbindlichkeit der Studierenden war schließlich das beste Mittel, um manche anfängliche Skepsis verfliegen zu lassen.

Nicht alle, aber fast alle Studierenden konnten den Master erfolgreich beenden; die meisten haben ein Doktoratsstudium angeschlossen. Sie mussten mit denselben Schwierigkeiten fertig werden, die auch universitäre Masterstudierende beim Abschluss beschäftigen: Wie wird aus einem vagen Interesse eine Forschungsfrage? Wie kann ich Methoden für deren Beantwortung finden? Nur haben diese Studierenden Dank ihrer künstlerischen Erfahrung und Ausbildung auch eigenes Wissen und Können, eigene Erfahrungen und Kompetenzen mitgebracht, um Methoden zu erarbeiten und nach Antworten zu suchen. Wissenschaft besteht - genauso wie Kunst - zu einem Teil aus Handwerk. Wer will, kann es lernen. Inspiration, Glück und Leidenschaft braucht's schließlich überall, sowohl an Universitäten als auch in der Kunst; darin unterscheiden sich die beiden Welten nicht. 